Engineering Physics and Mathematics Division

Mathematical Sciences Section

\title{
QMRPACK AND APPLICATIONS
}

\author{
Roland W. Freund $\dagger$ \\ Noël M. Nachtigal $\ddagger$ \\ $\dagger$ AT\&T Bell Laboratories \\ Room 2C-420 \\ 600 Mountain Avenue \\ Murray Hill, NJ 07974-0636 \\ freund@research.att.com \\ $\ddagger$ Mathematical Sciences Section \\ Oak Ridge National Laboratory \\ P.O. Box 2008, Bldg. 6012 \\ Oak Ridge, TN 37831-6367 \\ santa@msr.epm.ornl.gov
}

Date Published: October, 1994

Research was supported in part by the Applied Mathematical Sciences Research Program of the Office of Energy Research, U.S. Department of Energy and by the Research Institute for Advanced Computer Science (RIACS), NASA Ames Research Center, Moffett Field.

\author{
Prepared by the \\ Oak Ridge National Laboratory \\ Oak Ridge, Tennessee 37831 \\ managed by \\ Martin Marietta Energy Systems, Inc. \\ for the \\ U.S. DEPARTMENT OF ENERGY \\ under Contract No. DE-AC05-84OR21400
}




\section{DISCLAIMER}

This report was prepared as an account of work sponsored by an agency of the United States Government. Neither the United States Government nor any agency thereof, nor any of their employees, make any warranty, express or implied, or assumes any legal liability or responsibility for the accuracy, completeness, or usefulness of any information, apparatus, product, or process disclosed, or represents that its use would not infringe privately owned rights. Reference herein to any specific commercial product, process, or service by trade name, trademark, manufacturer, or otherwise does not necessarily constitute or imply its endorsement, recommendation, or favoring by the United States Government or any agency thereof. The views and opinions of authors expressed herein do not necessarily state or reflect those of the United States Government or any agency thereof. 


\section{DISCLAIMER}

Portions of this document may be illegible in electronic image products. Images are produced from the best available original document. 


\section{Contents}

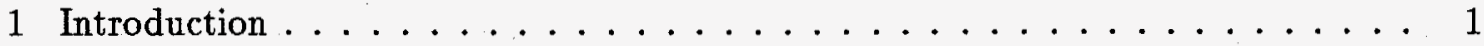

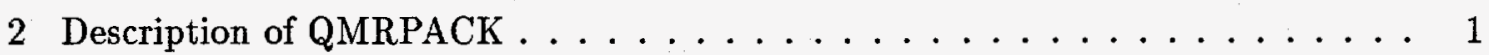

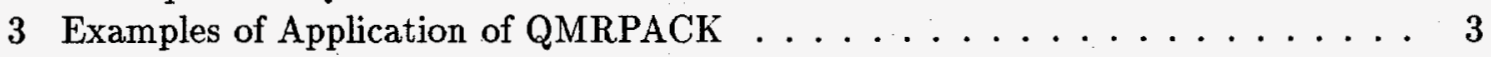

4 Future Directions . . . . . . . . . . . . . . . . . . . . . 4

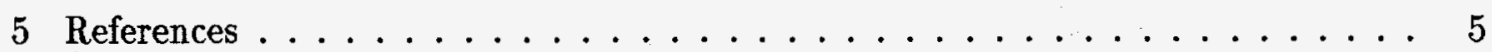





\title{
QMRPACK AND APPLICATIONS
}

\author{
Roland W. Freund \\ Noël M. Nachtigal
}

\begin{abstract}
QMRPACK is a package of quasi-minimal residual algorithms for the iterative solution of linear systems. In this paper, we describe the main features of QMRPACK, comment on the implementations found in the package, and discuss how they would be invoked in a user code. We briefly describe two applications of the codes in the area of simulation of material properties from first principles, and in circuit simulation. We conclude by presenting future directions for QMRPACK.
\end{abstract}




\section{Introduction}

Since the introduction of the quasi-minimal residual (QMR) algorithm for complex symmetric linear systems by Freund [3], much work has gone into developing more general and robust QMR methods. In [6], we extended the original algorithm to the case of general non-Hermitian matrices. The resulting algorithm uses the look-ahead variant [5] of the classical three-term Lanczos process [11] as the underlying method for building the Krylov basis. In $[7,8]$, we developed a coupled two-term recurrence variant of the look-ahead Lanczos process [7,8], where the three-term recursions are replaced by coupled two-term recursions that retain look-ahead but in practice have better numerical properties. In [4], Freund introduced the transpose-free QMR method (TFQMR), which eliminates the need for the transpose, but did not include any look-ahead and thus is susceptible to breakdowns. Work is in progress on a look-ahead variant of TFQMR that will allow look-ahead steps of limited sizes.

While the underlying look-ahead algorithms are relatively simple to explain at a high level, they are non-trivial to implement robustly. As a result, in 1990, we started a project to produce portable FORTRAN-77 codes implementing the various QMR algorithms as black-box solvers. The result is QMRPACK, a software package containing several QMR methods, as well as an eigenvalue solver.

\section{Description of QMRPACK}

QMRPACK is a collection of FORTRAN-77 library routines implementing several of the QMR algorithms and an eigenvalue solver, available from NETLIB. The package is distributed in the form of the compressed tar file "qmrpack.tar.Z" in the "linalg" subdirectory of NETLIB. It is only retrievable via: xnetlib or anonymous ftp. In the latter case, ftp to "netlib.att.com" and then get the file "qmrpack.tar.Z" from the directory "netlib/linalg".

The QMRPACK package currently includes three main QMR algorithms for the solution of square linear systems:

- The original QMR algorithm [6], based on the look-ahead three-term Lanczos process described in [5];

- The QMR algorithm based on the look-ahead coupled two-term recurrence Lanczos process $[7,8]$

- The TFQMR algorithm [4].

Even though the main emphasis of the package is on the linear systems solvers, also included is a routine for obtaining approximate eigenvalues from the look-ahead three-term Lanczos algorithm. In addition, the package includes simplified no-look-ahead versions of both the three-term and the coupled two-term QMR algorithms, as well as an implementation of the QMR-from-BCG algorithm proposed by Freund and Szeto [9]. The, codes 
are available for general non-Hermitian matrices, in single and double precision, real and complex data types. We will not describe here any of the algorithms in the package; full details can be found in the corresponding references. Although QMRPACK contains no-look-ahead variants of the QMR codes, this was done in the interest of completeness and with the understanding that the look-ahead variants are the more robust versions and their use is recommended over their no-look-ahead counterparts.

The distribution for QMRPACK includes the solvers, library support routines, example drivers, and example data formats and preconditioners. The library support routines include the BLAS routines, routines from LINPACK, and, for the eigenvalue solver, routines from EISPACK. The example drivers are intended to demonstrate the use of the black-box solvers, and include an example data format (the compressed sparse row format) and several preconditioners for this data format: right, left, and two-sided variants of the ILUT preconditioner proposed by Saad, and right, left, and two-sided variants of the SSOR preconditioner. We note in passing that the two-sided SSOR preconditioner is implemented using the Eisenstat trick, and thus the application of the preconditioned matrix is only marginally more expensive than the application of the unpreconditioned matrix.

As QMRPACK is intended to be a portable library of codes, the low-level solvers are written in strict ANSI FORTRAN-77. Only the example drivers are not strictly ANSI FORTRAN-77; they use the "\$" format specifier to indicate to the output processor that it should not advance to the next line after displaying a string. This format is supported by most FORTRAN compilers today (in fact, by all the compilers encountered by the authors), but strictly speaking, it is not ANSI FORTRAN-77.

Some of the issues that come up in the design and use of iterative methods solvers are the choice of a convergence criterion, the integration of the preconditioner in the algorithm, and the implementation of the matrix-vector operations. In QMRPACK, the convergence criterion used is the relative residual norm, $\left\|r_{n}\right\|_{2} /\left\|r_{0}\right\|_{2}$. This choice is hard-coded in the algorithms, and there is no facility for the user to change it without additional coding. We are aware that this is not the only choice available for convergence criteria. The preconditioner is not explicitly incorporated into the linear system solvers, though of course this could be done for all the algorithms in the package. The application of the preconditioner rests with the user, inside the matrix-vector routines, and the codes solve the alreadypreconditioned system (in fact, the codes further assume that the starting guess is always $x_{0}=0$ ). This means that all the quantities generated by the codes belong to the preconditioned system, and not the original system; in particular, the convergence criterion will use the preconditioned residual (unless right preconditioning is used) to determine convergence. For the matrix-vector routines, QMRPACK uses reverse communication. This mechanism consists of having the solver routines return to the caller, with a flag set, when a matrix-vector multiplication is needed, then having the caller perform the operation and call back the solver. The advantage of reverse communication is that it allows complete flexibility over the data structure for the matrix and the implementation 
of the matrix-vector operations, since all the matrix information is external to the solvers. The example drivers demonstrate how to set up the calls to the iterative solvers, how to recover the iterates of the original system from those returned by the routines, and how to handle the reverse communication protocol.

Finally, the routines in QMRPACK have several control parameters, allowing the user complete control over such aspects of the algorithm as the generation of the auxiliary starting vectors, the choice between the computation of the true residual norm at every step or the use of the residual norm upper bounds that are available in the QMR algorithms, output history, and so forth. We should also mention that all the vector operations in QMRPACK are implemented via calls to BLAS routines, which means that the package will benefit from optimized versions of these routines where available. It also means that porting the package to parallel architectures is fairly straightforward; indeed, codes from one of the earlier versions of QMRPACK have been ported to the Thinking Machines CM-5 and incorporated into the CMSSL scientific library.

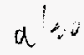

\section{Examples of Application of QMRPACK}

Nachtigal and Shelton (Oak Ridge National Laboratory) have recently incorporated several QMR algorithms from QMRPACK into codes for the computation of the electronic structure of materials using an approach based on first principles. At the heart of these computations is the explicit inversion of a matrix. The operation is repeated at many points on a grid, and many times in a self-consistent loop, leading to thousands or possibly millions of matrix inversions; this is the most time-consuming part of the computation. Previous versions of the codes used a direct approach to compute the inverses (either Gauss-Jordan or an LU decomposition). The new version replaces the direct solver with an iterative solver from QMRPACK. Preliminary results indicate that the new approach is significantly faster than the old one and that it scales much better as well. Figure 1 shows average times of a computation for Molybdenum, where TFQMR and an LU solver were used. Note that TFQMR and LU perform comparably if the cluster size is 9 atoms, but the TFQMR solver becomes significantly faster as the cluster size is increased and reaches a factor of 11 speedup in CPU time for a cluster size of 113 atoms. The difference is even more pronounced in user time, where the TFQMR solver is more than 40 times faster, as the direct solver codes are swapped out more often. More importantly, the tests have shown that the solution time increases linearly with the cluster size when the TFQMR solver is used, as opposed to cubic growth for LU. Thus, the new approach is shown to have significant potential both to speed up the computation and to extend it to larger cluster sizes than those previously attainable.

As a second example of an application of QMRPACK, we mention the recent work of Feldmann and Freund [2] who use the look-ahead Lanczos algorithm from QMRPACK to compute Padé approximations to the impulse-response functions of large linear electronic circuits. The resulting algorithm is called PVL (Padé approximation Via the look-ahead 


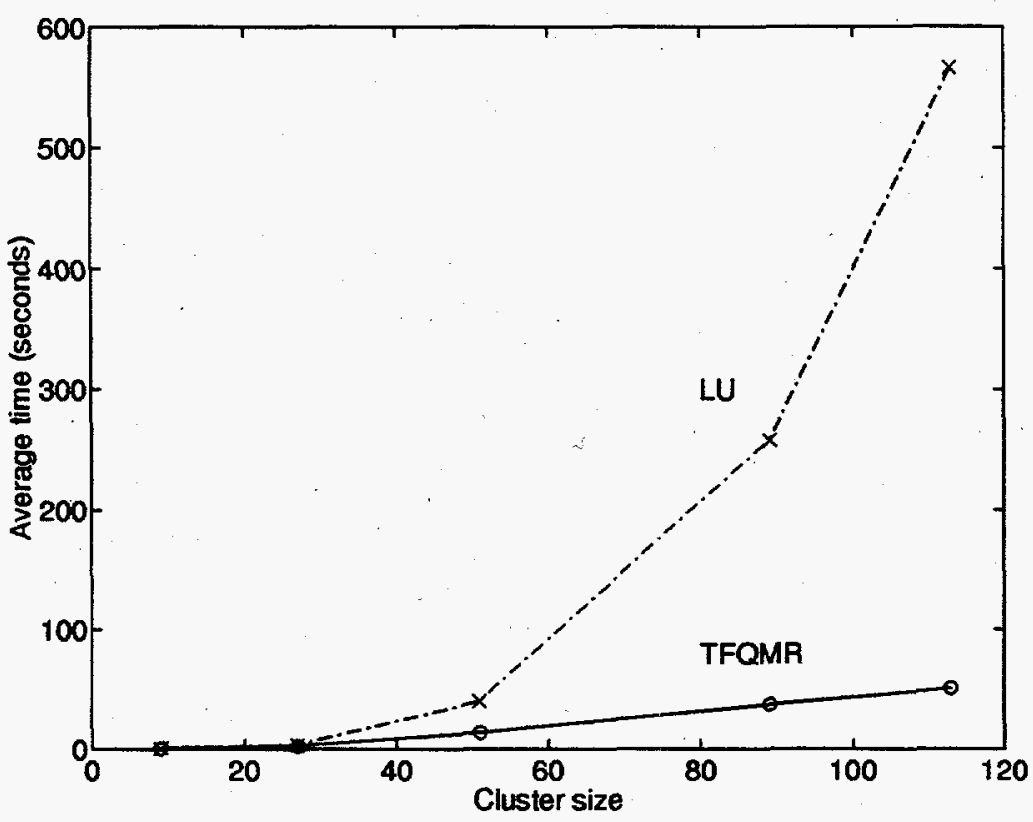

Figure 1: Average times for LU and TFQMR

Lanczos process). We illustrate the behavior of the PVL algorithm with one typical example taken from [2]. In Figure 2, we show the exact impulse response (solid line) and the approximation obtained after 60 Lanczos steps (dash-dotted line) for the PEEC circuit, which consists of 2100 capacitors, 172 inductors, and 6990 inductive couplings. Notice the excellent agreement of the two curves over a wide frequency range.

\section{Future Directions}

The next addition to QMRPACK will be codes for symmetric matrices, for which the underlying Lanczos algorithms simplify considerably, reducing work and storage requirements by a factor of two [3,10]. Of particular interest in this class are real symmetric indefinite matrices, and complex symmetric matrices. For real symmetric indefinite matrices, the QMR algorithms have the advantage of allowing the use of a symmetric indefinite preconditioner, while some of the other methods for symmetric indefinite systems, such as MINRES and SYMMLQ, require a symmetric positive definite preconditioner. For complex symmetric matrices, QMR has already been used quite successfully in industry for several years (see e.g. [1]), and the inclusion of codes for this class of matrices in QMRPACK will make them widely available.

As already indicated, work is in progress on a variant of TFQMR that would allow limited look-ahead. Preliminary results indicate that the new method is more robust than the original TFQMR algorithm, and an implementation of this method will be included 


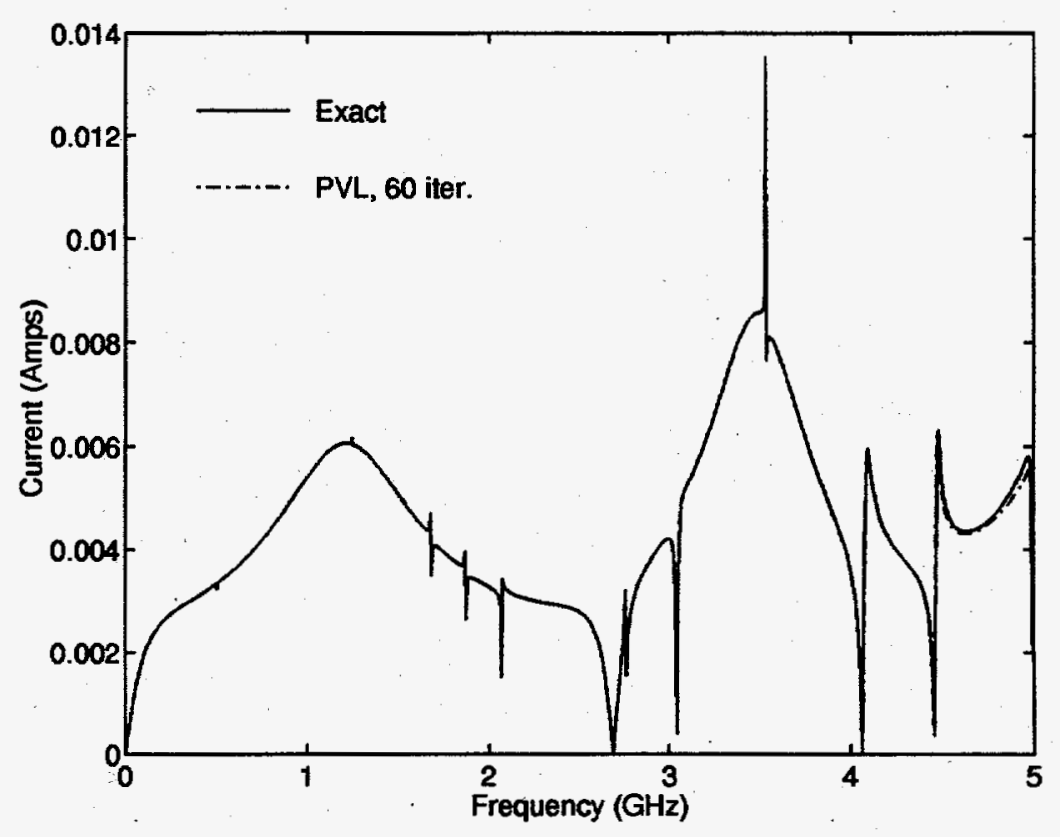

Figure 2: Simulation of the PEEC circuit, 60 PVL steps

in QMRPACK once the development is complete.

Finally, Freund has recently proposed a block Lanczos algorithm that would be suitable for use in the solution of linear systems with multiple right-hand sides. When the development is complete, we expect to incorporate codes implementing QMR algorithms based on the block Lanczos algorithm in QMRPACK As opposed to the rest of the codes already in QMRPACK and those mentioned above, we expect that the block variants of QMR will be coded in C, rather than FORTRAN. We also expect to investigate a distributed-computing version of this block code, based on PVM, and if successful, to include it in the package.

\section{References}

[1] W.E. Boyse and A.A. Seidl, "A sparse matrix iterative method for efficiently computing multiple simultaneous solutions", Manuscript, March 1994.

[2] P. Feldmann and R.W. Freund, "Efficient linear circuit analysis by Padé approximation via the Lanczos process", Numerical Analysis Manuscript 94-01, AT\&T Bell Laboratories, Murray Hill, NJ, 1994.

[3] R.W. Freund, "Conjugate gradient-type methods for linear systems with complex symmetric coefficient matrices", SIAM J. Sci. Statist. Comput. 13, pp. 425-448, 1992. 
[4] R.W. Freund, "A transpose-free quasi-minimal residual method for non-Hermitian linear systems", SIAM J. Sci. Comput. 14, pp. 425-448, 1993.

[5] R.W. Freund, M.H. Gutknecht, and N.M. Nachtigal, "An implementation of the look-ahead Lanczos algorithm for non-Hermitian matrices", SIAM J. Sci. Comput. 14, pp. 137-158, 1993.

[6] R.W. Freund and N.M. Nachtigal, "QMR: a quasi-minimal residual method for nonHermitian linear systems", Numer. Math. 60, pp. 315-339, 1991.

[7] R.W. Freund and N.M. Nachtigal, "Implementation details of the coupled QMR algorithm", Numerical Linear Algebra (L. Reichel, A. Ruttan, and R.S. Varga, eds.), pp. 123-140, W. de Gruyter, Berlin, 1993.

[8] R.W. Freund and N.M. Nachtigal, "An implementation of the QMR method based on coupled two-term recurrences", SIAM J. Sci. Comput. 15, pp. 313-337, 1994.

[9] R.W. Freund and T. Szeto, "A transpose-free quasi-minimal residual squared algorithm for non-Hermitian linear systems", Advances in Computer Methods for Partial Differential Equations - VII (R. Vichnevetsky, D. Knight, and G. Richter, eds.), pp. 258-264, IMACS, 1992.

[10] R.W. Freund and H. Zha, "Simplifications of the nonsymmetric Lanczos process and a new algorithm for Hermitian indefinite linear systems", AT\&T Numerical Analysis Manuscript, Bell Laboratories, Murray Hill, NJ, 1994.

[11] C. Lanczos, "An iteration method for the solution of the eigenvalue problem of linear differential and integral operators", J. Res. Nat. Bur. Standards 45, pp. 255-282, 1950 . 
ORNL/TM-12753

\section{INTERNAL DISTRIBUTION}

1. B.R. Appleton

2-3. T.S. Darland

4. E.F. D'Azevedo

5. J.M. Donato

6. J.J. Dongarra

7. G.A. Geist

8. S.L. Lee

9. M.R. Leuze

10-14. E.G. $\mathrm{Ng}$

15. C.E. Oliver

16. P.M. Papadopoulos

17-21. S.A. Raby
22. C.H. Romine

23. T.H. Rowan

24. B.D. Semeraro

25. W.A. Shelton

26-30. R.F. Sincovec

31-35. R.C. Ward

36. Central Research Library

37. ORNL Patent Office

38. K-25 Appl Tech Library

39. Y-12 Technical Library

40. Lab Records Dept - RC

41-42. Laboratory Records Dept

\section{EXTERNAL DISTRIBUTION}

43. Loyce Adams, Dept. of Applied Mathematics, FS-20, University of Washington, Seattle, WA 98195

44. Greg Ammar, Dept. of Mathematical Sciences, Northern Illinois University, DeKalb, IL 60115

45. Steven Ashby, Lawrence Livermore National Lab, P.O. Box 808, M/S L-316, Livermore, CA 94551-0808

46. Donald M. Austin, 6196 EECS Bldg., University of Minnesota, 200 Union St., S.E., Minneapolis, MN 55455

47. Owe Axelsson, Faculty of Mathematics and Informatics, University of Nijmegen, NL6525 ED Nijmengen The Netherlands

48. Jesse Barlow, Dept. of Camputar Science and Engineering, Pennsylvania State Uri versity, 220 Pond Laboratory, University Park, PA 16802-6106

49. Edward H. Barsis, Sandia National Laboratories, Computer Science and Mathematics, P.O. Box 5800, Albuquerque, NM 87185

50. Ake Bjorck, Dept. of Mathematics, Linkoping University, S-58183 Linkoping, Sweden

51. Petter Bjorstad, Institutt for Informatikk, Hoyteknologisenteret, N-5020 Bergen, Norway

52. Roger W. Brockett, Harvard University, 29 Oxford Street, Cambridge, MA 021382901

53. Richard Brualdi, Dept. of Mathematics, 480 Lincoln Drive, Madison, WI 53706

54. Angelika Bunse-Gerstner, Fachbereich Mathematik und Informatik, Universität Bremen, Postfach 3304 40, D-28334 Bremen, Federal Republic of Germany 
55. Daniela Calvetti, Stevens Institute of Technology, Dept. of Mathematics, Hoboken, NJ 07030

56. Ian Cavers, Dept. of Computer Science, University of British Columbia, Vancouver, British Columbia V6T 1W5, Canada

57. Tony Chan, Dept. of Mathematics, University of California, Los Angeles, 405 Hilgard Avenue, Los Angeles, CA 90024-1555

58. Paul Concus, Mathematics and Computing, Lawrence Berkeley Laboratory, 50A/2129; Berkeley, CA 94720

59. Jane Cullum, IBM T.J. Watson Research Center, P.O. Box 218, Yorktown Heights, NY 10598

60. Biswa Datta, Dept. of Mathematical Sciences, Northern Illinois University, DeKalb, IL 60115

61. James Demmel, Dept. of Computer Science, University of California, 571 Evans Hall, Berkeley, CA 94720

62. John Dorning, Dept. of Nuclear Engineering Physics, Nuclear Reactor Facility, University of Virginia, Charlottesville, VA 22903-2442

63. Donald J. Dudziak, Dept. of Nuclear Engineering, 110B Burlington Engineering Labs, North Carolina State University, Raleigh, NC 27695-7909

64. Michael Eiermann, Institut für Angewandte Mathematik II, TU Bergakademie Freiberg, D-09596 Freiberg, Federal Republic of Germany

65. Howard Elman, Dept. of Computer Science, University of Maryland, College Park, MD 20742

66. Robert Ferraro, Jet Propulsion Lab, M/S 198-136A, 4800 Oak Grove Drive, Pasadena, CA 91109

67. Bernd Fischer, Universität Hamburg, Institut fur Angewandte Mathematik, Bundersstrasse 55, D-2000 Hamburg 13, Federal Republic of Germany

68. Geoffrey C. Fox, Northeast Parallel Architectures Center, 111 College Place, Syracuse University, Syracuse, NY 13244-4100

69 Efstratios Gallopoulos, CSRD, 465 C \& SRL, University of Illinois, 1308 W Main Street, Urbana, IL 61801-2983

70. J. Alan George, Vice President, Academic and Provost, Needles Hall, University of Waterloo, Waterloo, Ontario, Canada N2L 3G1

71. Gene H. Golub, Dept. of Computer Science, Stanford University, Stanford, CA 94305

72. William Gragg, Dept. of Mathematics, Naval Postgraduate School, Monterey, CA 93943-5100

73. Joe Grcar, Sandia National Laboratories, Division 8245, Livermore, CA 94551-0969

74. Anne Greenbaum, Courant Institute, New York University, 251 Mercer Street, New York, NY 10012-1185

75. John Gustafson, Ames Laboratory, Iowa State University, Ames, IA 50011

76. Martin Gutknecht, IPS Supercomputing, ETH-Zentrum, CH-8092 Zurich, Switzerland 
77. Christian Halloy, Assistant Director of JICS, 104 South College, Joint Institute for Computational Science, University of Tennessee, Knoxville, TN 37996-1301

78. Martin Hanke, Universität Karlsruhe, Institut fur Praktische Mathematik, Englerstrasse 2, D-76128 Karlsruhe, Federal Republic of Germany

79. Michael Heroux, Cray Research Inc, 655F Lone Oak Dr, Eagan, MN 55121-1560

80. Nicholas Higham, Dept. of Mathematics, University of Manchester, Grt Manchester, M13 9PL, England

81. Marlis Hochbruck, Mathematisches Institut, Universität Tuebingen, Auf der Morgenstelle 10, D-72076 Tuebingen, Federal Republic of Germany

82. Louis Howell, Lawrence Livermore National Lab, P.O. Box 808, M/S L-316, Livermore, CA 94550-0808

83. Ilse Ipsen, Dept. of Mathematics, North Carolina State University, Raleigh, NC 27695-8205

84. Wayne Joubert, Los Alamos National Lab, Group C3, M/S B-265, Los Alamos, NM 87545

85. Malvyn H. Kalos, Cornell Theory Center, Engineering and Theory Center Bldg., Cornell University, Ithaca, NY 14853-3901

86. Hans Kaper, Mathematics and Computer Science Division, Argonne National Laboratory, 9700 South Cass Avenue, Bldg. 221, Argonne, IL 60439

87. Herbert Keller, Dept. of Applied Mathematics, 217-50, California Institute of Technology, Pasadena, CA 91125

88. Kenneth Kennedy, Dept. of Computer Science, Rice University, P.O. Box 1892, Houston, TX 77001

89. David Keyes, NASA Langley Research Center, ICASE, M/S L23C, Hampton, CA 23681-0001

90. Frank Korsmeyer, Dept. of Ocean Engineering, 5-326, Massachusetts Institute of Technology, Cambridge, MA 02139

91. Richard Lehoucq, Dept. of Computational and Applied Mathematics, P.O. Box 1892, Rice University, Houston, TX 77251-1892

92. James E. Leiss, Rt. 2, Box 142C, Broadway, VA 22815

93. John G. Lewis, Boeing Computer Services, P.O. Box 24346, M/S 7L-21, Seattle, WA 98124-0346

94. Eric Lucas, 8787 Manahan Drive, Ellicott City, MD 21043

95. Thomas A. Manteuffel, Program in Applied Mathematics, Campus Box 526, University of Colorado, Boulder, CO 80309-0526

96. Kapil Mathur, Thinking Machines Corporation, 245 First Street, Cambridge, MA 02142

97. James McGraw, Lawrence Livermore National Laboratory, P.O. Box 808, M/S L-306 Livermore, CA 94550

98. Cleve Moler, The Mathworks, 24 Prime Park Way, Natick, MA 01760 
99. Neville Moray, Dept. of Mechanical and Industrial Engineering, University of Illinois, 1206 West Green Street, Urbana, IL 61801

100. David Nelson, Director of Scientific Computing, ER-7, Applied Mathematical Sciences, Office of Energy Research, U.S. Dept. of Energy, Washington, DC 20585

101. Wilhelm Niethammer, Universität Karlsruhe, Institut fur Praktische Mathematik, Englerstrasse 2, D-76128 Karlsruhe, Federal Republic of Germany

102. Dianne P. O'Leary, Dept. of Computer Science, University of Maryland, College Park, MD 20742

103. James M. Ortega, Dept. of Applied Mathematics, Thornton Hall, University of Virginia, Charlottesville, VA 22901

104. Elizabeth Ong, Dept. of Mathematics, 9500 Gilman Drive, University of California, La Jolla, CA 92093-0112

105. Michael Overton, Courant Institute, New York University, 251 Mercer Street, New York, NY 10012-1185

106. Beresford N. Parlett, Dept. of Mathematics, University of California, Berkeley, CA 94720

107. Alexander Peters, IBM Corporation, Heidelberg Scientific Center, Vangerowstrasse 18, D-69020 Heidelberg, Federal Republic of Germany

108. Linda Petzold, Dept. of Computer Science, University of Minnesota, 4-192 EE/CS Bldg, 200 Union Street S.E., Minneapolis, MN 55455-0159

109. Robert J. Plemmons, Dept.s of Mathematics and Computer Science, Box 7311, Wake Forest University, Winston-Salem, NC 27109

110. Jame. C. T. Pool, Deputy Director, Caltech Concurrent Supercomputing Facility, California Institute of Technology, Pasadena, CA 91125

111. Jesse Poore, Dept. of Computer Science, Ayres Hall, University of Tennessee, Knoxville, TN 37996-1301

112. Lothar Reichel, Dept. of Mathematics, Kent State University, Kent, OH 44242

113. Werner C. Rheinboldt, Dept. of Mathematics and Statistics, University of Pittsburgh, Pitisburgh, PA 15260

114. Youcef Saad, Dept. of Computer Science, University of Minnesota, 4-192 EE/CS Bldg, 200 Union Street S.E., Minneapolis, MN 55455-0159

115. Ahmed H. Sameh, Dept. of Computer Science, University of Minnesota, 4-192 EE/CS Bldg, 200 Union Street S.E., Minneapolis, MN 55455-0159

116. Michael Saunders, Systems Optimization Laboratory, Operations Research Dept., Stanford University, Stanford, CA 94305

117. Robert Schreiber, RIACS, Mail Stop 230-5, NASA Ames Research Center, Moffet Field, CA 94035

118. David S. Scott, Intel Scientific Computers, 15201 N.W. Greenbrier Parkway, Beaverton, OR 97006

119. Valeria Simoncini, Via Pacinotti 10, 40024 Castel S Pietro, Bologna, Italy 
120. Horst Simon, Mail Stop T045-1, NASA Ames Research Center, Moffett Field, CA 94035

121. Danny Sorensen, Dept. of Mathematical Sciences, Rice University, P.O. Box 1892, Houston, TX 77251

122. Gerhard Starke, Universität Karlsruhe, Institut fur Praktische Mathematik, Englerstrasse 2, D-76128 Karlsruhe, Federal Republic of Germany

123. Zdenek Strakos, Czech Academy of Sciences, Pod vod.vezi 2, 18208 Praha 8, Czech Republic

124. Paul N. Swartztrauber, National Center for Atmospheric Research, P.O. Box 3000, Boulder, CO 80307

125. Daniel Szyld, Dept. of Mathematics, Temple University, TU038-16, Philadelphia, PA $19122-2585$

126. Wei Pai Tang, Dept. of Computer Science, University of Waterloo, Waterloo N2L 3G1, Ontario, Canada

127. Nick Trefethen, Dept. of Computer Science, Cornell University, Upson Hall, Ithaca, NY 14853

128. Raymond S. Tuminaro, Sandia National Laboratories, Mail Stop 1110, P.O. Box 5800, Albuquerque, NM 87185

129. Hank Van der Vorst, Dept. of Techn. Mathematics and Computer Science, Delft University of Technology, P.O. Box 356, NL-2600 AJ Delft, The Netherlands

130. Paul Van Dooren, Dept. of Electrical and Computer Engineering, University of Illinois, 1101 W. Springfield Avenue, Urbana, IL 61801

131. Charles Van Loan, Dept. of Computer Science, Cornell University, Ithaca, NY 14853

132. Jim Varah, Centre for Integrated Computer Systems Research, University of British Columbia, Office 2053-2324 Main Mall, Vancouver, British Columbia V6T 1W5, Canada

133. Madan Verma, Dept. of Electrical Engineering, 3480 University Street, McGill University, Montreal H3A 2A7, Quebec, Canada

134. Phuong Vu, Cray Research, Inc., 19607 Franz Road, Houston, TX 77084

135. Mary F. Wheeler, Rice University, Dept. of Mathematical Sciences, P.O. Dor 1892 . Houston, TX 77251

136. Andrew B. White, Computing Division, Los Alamos National Laboratory, P.O. Box 1663, MS-265, Los Alamos, NM 87545

137. Jacob White, Dept. of Electrical Engineering and Computer Science, Massachusetts Institute of Technology, Room 36-880, 50 Vassar Street, Cambridge, MA 02139-4309

138. David Young, Boeing Computer Services, M/S 7L-21, P.O. Box 24346, Seattle, WA 98124-0346

139. David Young, University of Texas, Center for Numerical Analysis, RLM 13.150, Austin, TX 78731-8510

140. Office of Assistant Manager for Energy Research and Development, U.S. Dept. of Energy, Oak Ridge Operations Office, P.O. Box 2001 Oak Ridge, TN 37831-8600

141-142. Office of Scientific \& Technical Information, P.O. Box 62, Oak Ridge, TN 37831 